\title{
Biologically Inspired Bio-Cyber Interface Architecture and Model for Internet of Bio-NanoThings Applications
}

Uche A.K. Chude-Okonkwo, Reza Malekian and B.T. Maharaj

Abstract-With the advent of nanotechnology, concepts related to the Internet of Things, such as the Internet of NanoThings (IoNT) and Internet of Bio-NanoThings (IoBNT) have also emerged in the classical literature. Of concern in this paper is the IoBNT, which projects the prospective application domain where the activities of very tiny, biocompatible, and non-intrusive devices operating in an in-body nanonetwork can be monitored and controlled through the Internet. In this paper, we present an illustrative scenario and system model of an IoBNT for application in an advanced healthcare delivery system. To address one of the major challenges of the IoBNT, we present an exemplary architecture and model of a bio-cyber interface for connecting the conventional electromagnetic-based Internet to the biochemical signaling-based bionanonetwork. The bio-cyber interface is designed and modeled by employing biological concepts such as the responsiveness of certain biomolecules to thermal and light stimuli, and the bioluminescence phenomenon of some biochemical reactions. The analysis in this paper focuses on the system that comprises the bio-cyber interface and the information propagation network of the blood vessel that leads to the in-body nanonetwork location. The effects of the system and design parameters associated with the IoBNT models presented are numerically evaluated.

Index Terms - Internet of bio-nanothings, nanosensor, molecular communication, nanodevices, nanotechnology 


\section{INTRODUCTION}

Nanotechnology has ushered in ideas, tools and devices with the potential to improve existing technologies as well as introduce entirely new scientific innovations. This technology dwells on the concept of manipulating materials at the particulate and molecular levels of systems. A branch of nanotechnology that has attracted great attention in recent time is molecular communication (MC) [1]. MC is a new communication paradigm that uses biochemical signals to achieve information exchange among naturally and artificially created bio-nano scale devices over short distances [2-5]. It has found potential application in the field of medicine, where subjects such as targeted drug delivery (TDD) [6-8] and disease diagnosis/monitoring/therapy [9] executed at the nanoscale level have been considered. A review of the application of MC to healthcare delivery can be found in [10].

In the TDD scenario, the injection and transportation of drug particles in the human body and delivery to a specific tissue or organ can be viewed in the context of MC. In this sense, the drug particle injection device is the transmitter, the drug particles are information carriers, the blood vessel network is the communication channel and the targeted tissue forms part of the receiver $[6,7]$. In the case of disease diagnosis, monitoring and therapy, nanodevices can be used as analytic and imaging tools, biochemical sensors for monitoring, and tissue engineering tools for in vivo tissue repair and regeneration [11]. Technically, the tiny dimensions of these nanodevices and their biocompatible nature endear them as powerful tools for the manipulation of bio-materials at the nanoscale level of organisms and systems.

Conversely, the minute size of these devices limits their ability to take on complex tasks. Hence, many nanodevices will be required to work cooperatively in order to achieve a given task [12]. Some of the functionalities that these devices will possess include sensing/detection, capturing, storing, releasing and synthesizing biochemical molecules, acquiring and expending energy, moving and actuating, and replicating/terminating functioning [13]. The number of interconnected nanodevices in an intra-body nanonetwork will typically range from a few to millions. Therefore, ensuring efficient communication among these nanodevices is an important and very challenging task. In some cases, the capability of 
nanodevices in a given nanonetwork can be enhanced by connecting them to other networks, which may be another nanonetwork or a classical network. Such communication can also be enhanced by interconnecting the nanonetworks through the Internet in what is termed the Internet of Bio-nanothings (IoBNT) [14]. Indeed, the power and potential of any technology/tool/network are magnified when one starts combining it with other technologies/tools/networks. The IoBNT stands as novel paradigm-shifting concept for a prospective application domain where very tiny, biocompatible and non-intrusive devices can communicate through the Internet [15]. The IoBNT is the focus of this paper and has great potential to open up new possibilities in areas and applications where MC is envisaged to have great impact. The IoBNT application of concern in this work is the healthcare delivery system.

An illustration of the concept of IoBNT for healthcare application is shown in Fig. 1. Imagine an advanced medical therapeutic scenario, where a patient with a certain ailment is injected with a particular set of nanodevices that enter into some tissues to perform diagnostic activities, TDD or tissue repair. The patient is then allowed to go home and go about his/her normal duties while the nanodevices silently perform the designated tasks in the targeted system. The activities and progress of the nanodevices are then monitored by medical personnel at a remote location. The medical personnel receives/sends out signals/commands using conventional communicational terminals. The command the medical personnel sends may be some sets of binary codes, each instructing specific sets of nanodevices in the patient's body to execute functions such as replicate, move, sense, acquire/expend energy, release or synthesize molecules and terminate functioning/decompose. On the other hand, the signals/information the medical personnel receives will usually come from specific sets of nanodevices that act as nanosensors at the target nanonetwork inside the patient's body. Such information will reveal the states of the nanodevices and that of the microenvironment of the targeted tissues. It can tell the temperature, $\mathrm{pH}$, salinity and presence of certain biochemical compounds at a certain time. The information received can, among others, tell when a set of devices is no longer needed and should be eliminated from the system. This information can then be acted upon by the medical personnel to enhance the performance of the nanodevice network by sending 
appropriate commands for specific functions to be initiated.

In Fig. 1, to initiate a certain command, the medical personnel sends out the applicable signal through the Internet or any other appropriate network. This signal is received and relayed by a bio-cyber signaling system on the patient's body (for example on the wrist). The bio-cyber system responds by sending the appropriate signal to the designated set of devices in the target nanonetworks through the body channel, which can be the blood vessels. In our illustration in Fig. 1, the bio-cyber signal is received by a designated nanotransmitter in the targeted nanonetwork, which decodes the signal and sends out appropriate biochemical signals to the targeted nanodevices. The targeted nanodevices uniquely respond by executing the desired function. To allow for free movement of the patient and better signal reception, we have included an access point, which broadcasts the command received from the Internet.

There are many challenges facing the engineering and realization of a practical IoBNT. These challenges include those associated with the design and development of the nanodevices, the molecular communication within the nanonetwork and the interfacing between the nanonetwork and the Internet. Other challenges include network security and nanodevice tracking and localization [14]. Addressing these challenges are open problems in nanocommunication research.

Our main objective in this paper is to present a descriptive and system model of the IoBNT, and an example of an architecture/model of a bio-cyber interface. Specifically, we create an example of an IoBNT scenario and proceed to present a descriptive view of the scenario and the system model of the two-way communication between the medical personnel and the nanodevices in an intra-body nanonetwork. The scenario we consider in this work is illustrated in Fig. 1. This scenario depicts an IoBNT for TDD in which combination therapy [16] is employed for disease treatment. The term combination therapy defines the use

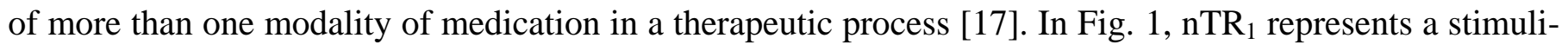
triggered liposome-based nanocarrier; $\mathrm{nTR}_{2}$ and $\mathrm{nTR}_{3}$ are nanodevices with the ability to synthesize different molecules when excited by extracellular signals. Hence, we can refer to $\mathrm{nTR}_{1}, \mathrm{nTR}_{2}$ and $\mathrm{nTR} \mathrm{TR}_{3}$ as nanotransceivers. We shall henceforth refer to the nanotransceivers and the nanosensor as nanodevices. 


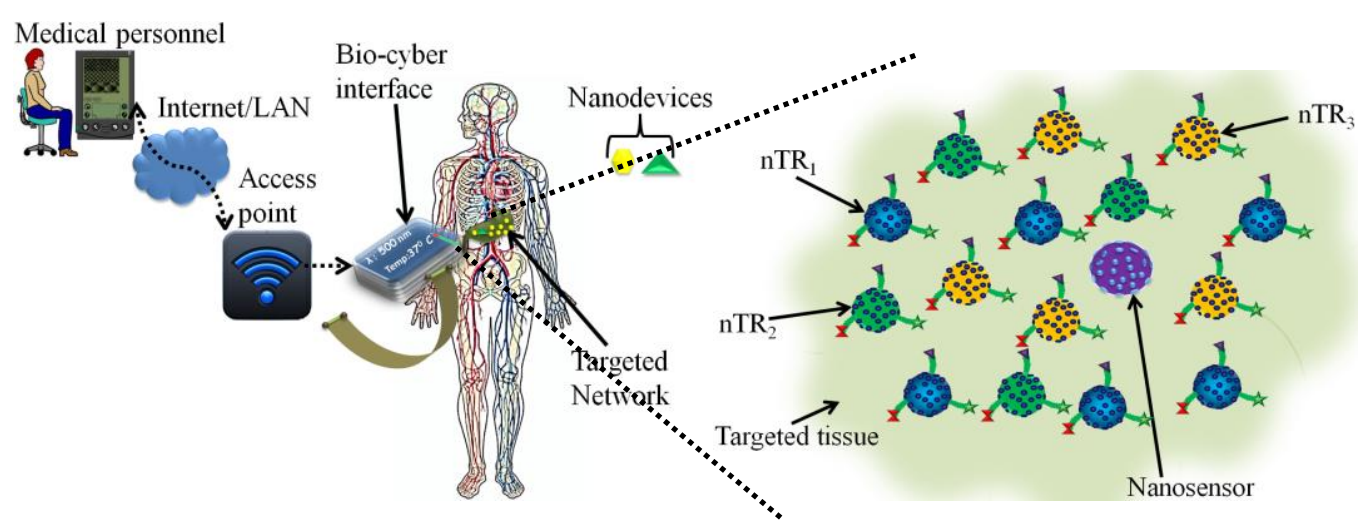

Fig. 1. Illustrative IoBNT system for TDD-based combination therapy.

In the scenario depicted above, a patient is injected with a set of therapeutic nanodevices, $\mathrm{nTR}_{1}, \mathrm{nTR}_{2}$, $\mathrm{nTR}_{3}$ and a nanosensor. The patient is then allowed to go home, while the nanodevices propagate through his blood vessel network to the targeted tissue. The nanodevices are equipped with specific ligands that can bind to complementary receptors that are only expressed at the targeted sites. The binding is a high-affinity one that keeps the nanodevices anchored at the site. At that point, certain enzymes that are specifically expressed at the targeted sites, such as matrix metalloproteinase (MMP) [18] in the case of a cancerous site, stimulate $n T R_{1}$ to release drug molecules, say $G_{A}$, which they encapsulate. The molecules released by $n T R_{1}$ are detected by the nanosensor in the targeted environment. The nanosensor responds to the presence of $\mathrm{G}_{\mathrm{A}}$ by emitting a certain molecule $\mathrm{G}_{\mathrm{B}}$, which propagates through the blood network and is detected by the biocyber interface located on the patient's wrist or any other convenient part of the body. The bio-cyber interface decodes the molecular information defined by $\mathrm{G}_{\mathrm{B}}$ and emits an appropriate electromagnetic-based signal. The emitted signal is received by the access point and is relayed through the Internet to the medical personnel. The information received by the medical personnel typically reports that the first therapeutic drug molecule $\mathrm{G}_{\mathrm{A}}$ has been delivered to the targeted site. Based on this information, the medical personnel sends a command to $\mathrm{nTR}_{2}$ to synthesize and emit the next therapeutic drug $\mathrm{G}_{\mathrm{C}}$. This command goes through the Internet to the bio-cyber interface, which responds by emitting a specific concentration of the molecule $G_{D}$ into the blood network. The molecules then traverse the blood network and reach the targeted site, where they trigger the synthesis and emission of $\mathrm{G}_{\mathrm{C}}$. When the drug delivery process has been completed, it is desirable that the nanodevices be eliminated from the body system. Hence, after the drug 
delivery process a death command is sent to $\mathrm{nTR}_{3}$ to excite all the nanodevices in the network to execute self-annihilation [15]. On receiving the death command, the $\mathrm{nTR}_{3}$ broadcasts a death signal, which the respective nanodevices receive; they then commit to self-annihilation.

In this paper, we present the system model of the IoBNT scenario presented above. The salient features of our work are as follows.

1) Theoretic model of IoBNT: By considering an illustrative IoBNT scenario for TDD, we present a descriptive and system model of an abstracted IoBNT. The abstracted system divides the IoBNT into the conventional Internet, the bio-cyber interface, the blood vessel network and the intrabody nanonetwork.

2) Example of model of a bio-cyber interface: The architecture and model of an example of a biocyber interface for the IoBNT scenario described in 1) is presented. The architecture and model are inspired by known biological phenomena. The interface input-output expressions are given.

3) Performance evaluation: The quantitative simulation and performance evaluation of the IoBNT subsystem that comprises the bio-cyber interface, the blood vessel network (using compartmental model) and the targeted in-body nanonetwork are provided.

The rest of this paper is organized as follows. The system model and problem definition are presented in Section II. In Section III, the bio-cyber interface architecture and model for the example of the IoBNT scenario are presented. The compartmental model for the propagation of a molecular signal through the blood vessel network to the nanonetwork location is presented in section IV. In Section V, the capacity of the IoBNT is discussed. The simulations and system performance analysis are provided in Section VI.

\section{SYSTEM MODEL}

A block diagram model of Fig. 1 is shown in Fig. 2(a). The different functional blocks in the communication sequence, namely Internet, access point, wireless channel, bio-cyber interface, blood network and targeted nanodevice, are designated as h1, h2, h3, h4, h5 and h6, respectively. As stated 
earlier, the command from the personnel's terminal will be a unique binary code that instructs the nanonetwork to execute certain functions. In our case, these functions include synthesizing and releasing molecules, sensing specific molecules and executing self-annihilation.

Let the time-varying responses of the Internet, access point, wireless channel, bio-cyber device, body channel and nanodevice be represented as $h_{1}(t), h_{2}(t), h_{3}(t), h_{4}(t), h_{5}(t)$ and $h_{6}(t)$, respectively. The model for $h_{1}(t)$ and $h_{2}(t)$ is the conventional Internet of things (IoT) model. Recently, the model for $h_{6}(t)$ in consideration of the elimination of nanodevices from a specified nanonetwork in a given IoBNT has been presented in $[15,19]$. In this present work, we specifically focus on the end-to-end physical layer system denoted by $h_{3}(t), h_{4}(t)$ and $h_{5}(t)$, as enclosed in the red dotted line in Fig. 2(b). Hence, for simplicity, we neglect the effect of errors and delays that arise in $h_{1}(t)$ and $h_{2}(t)$ and assume that the information transmitted by the access point is the appropriate command sent by the medical personnel. Thus, we can model the communication link through $h_{1}(t), h_{2}(t)$ and $h_{3}(t)$ as a wireless channel whose output $y^{(n)}(t)$ is

$$
y^{(\boldsymbol{n})}(t)=x^{(\boldsymbol{n})}(t) *\left(h_{1}(t) * h_{2}(t) * h_{3}(t)\right), \quad \boldsymbol{n}=\{f, r\}
$$

where $x^{(\boldsymbol{n})}(t)$ is the input signal, and ' $*$ ' denotes convolution operator. The superscript ${ }^{(n)}$ defines the direction of the communication, where $\left({ }^{(f)}\right.$ denotes communication from medical personnel to nanodevices, and ${ }^{(r)}$ denotes communication from nanosensors to medical personnel. A brief discussion of the nanodevices in this model, which include the nanotransceivers and nanosensor, is given below.

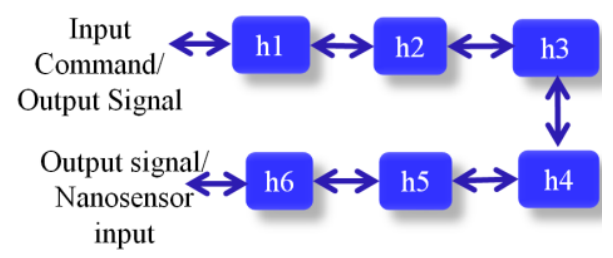

(a)

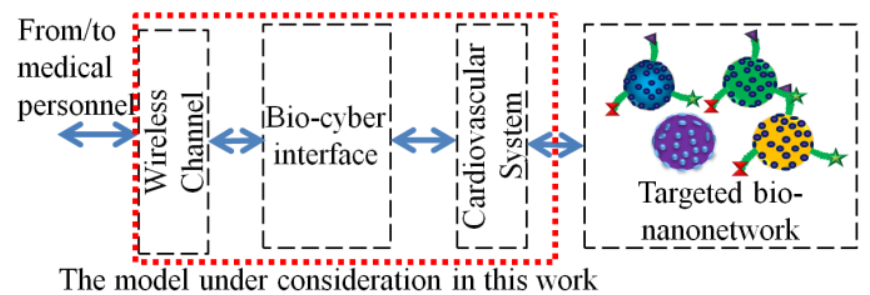

(b)

Fig. 2. (a) Schematic block diagram of the IoBNT scenario (b) Block diagram of the IoBNT subsystem considered in this work. 


\section{$n T R_{1}$}

This is a liposome that incorporates capabilities such as long circulation, stimuli responsiveness and targeting characteristics $[20,21]$. It is denoted as a transceiver here, since it releases encapsulated molecules when excited by external triggers. The $\mathrm{nTR}_{1}$, when injected into the vascular system, propagates through the blood vessel network and into the targeted site, where it anchors. Timely drug release by $\mathrm{nTR}_{1}$ at the targeted site requires that trigger stimuli for release, such as $\mathrm{pH}$, light and enzymes, be uniquely overexpressed at the targeted site. In this work, we assume that this is so, so that on reaching the targeted sites, the liposomes release their contents by virtue of the stimuli-triggered release.

\section{$n T R_{2} / n T R_{3}$}

These nanotransceivers are bionanodevices with the capability to synthesize and emit specific molecules. In the case of $\mathrm{nTR}_{2}$, the emitted molecules will be the second therapeutic drug molecules, while in nTR 3 , the emitted molecules will be the death-initiating molecules meant for the elimination of the devices from the system. The $n \mathrm{nR}_{2}$ and $\mathrm{nTR}_{3}$ can be achieved by the use of genetically engineered biological cells [22], a genetically engineered bacterium [23] and artificial cells [24]. In [25, 26] model examples of a genetically engineered biotransceiver that can be used to model the molecule synthesis and emission in nTR 2 and nTR3 are presented. The systems biology related model for the execution of self-annihilation in the nanodevices is presented in [15].

\section{Nanosensor}

This device is a bionanosensor, which must have the high sensitivity capable of detecting the presence of $\mathrm{G}_{\mathrm{B}}$ molecules and responding by emitting $\mathrm{G}_{\mathrm{C}}$. Such a nanosensor can be designed by using a whole-cell [27-29] or sensor molecules entrapped in a chemically inert matrix [30]. A mathematical model can be built for the nanosensor by taking into consideration the biochemical kinetics associated with its operation.

The nanodevices discussed above and the information molecules to and from the bio-cyber interface are typically conveyed to and from the targeted site through the cardiovascular system. In our work, we model the propagation of the molecular signals through the cardiovascular system using the compartmental model 
[31]. We shall proceed in the next sections to present models of the bio-cyber interface and the compartmental model for the blood vessel network.

\section{BIO-CYBER INTERFACE ARCHITECTURE AND MODEL}

The bio-cyber interface is a unit that processes and translates information from the Bio-NanoThing networks to the Internet cyber-domain, which is based on electrical circuits and electromagnetic communications and vice versa [14]. The design of a suitable interface that is able to interconnect the nanoscale biological environment with the external world is an open research topic in molecular communication and IoBNT [14]. The choice of an architecture for a bio-cyber interface will depend on the nature of the signal, the channel through which the signal in propagated and the task at hand. Some proposals to address the interface issue have been presented in [32-34].

In this paper, we desire to model a device that can decode a message carried by an electromagnetic wave and use the message to drive a system that produces a biochemical signal, and vice versa. A block diagram of such a system is depicted in Fig. 3, where $c^{(r)}(t)$ and $c^{(f)}$ are biological signals in and out of the bio-cyber interface, respectively. In the forward path, the electro-bio unit converts the electrical signal from the receiver unit to a biochemical output. In the reverse path, the bio-electro unit converts the biochemical signal transmitted by the bionanosensor and detected in the blood network to electrical output. The transmitter and receiver depicted in Fig. 3 are typically modeled like any conventional communication system. Hence, we shall concentrate on the models of the electro-bio and bio-electro transduction units.

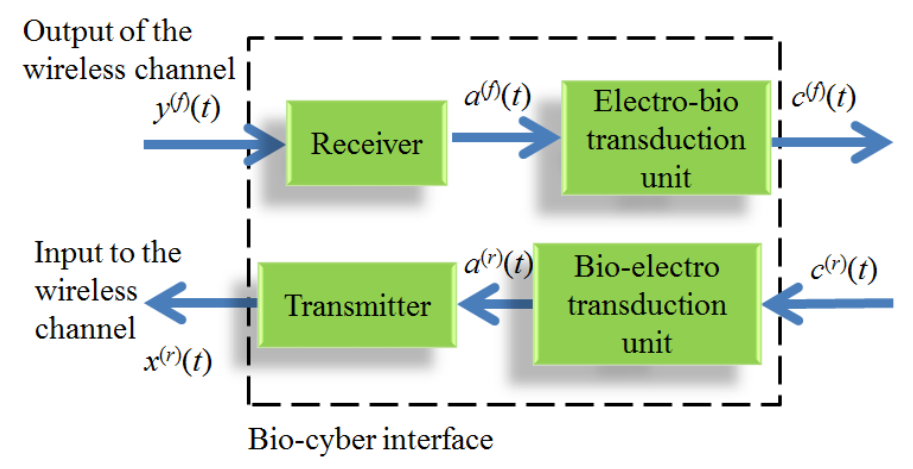

Fig. 3. Schematic block diagram of the bio-cyber interface. 


\section{A. Electro-bio interface model}

The architecture of the electro-bio transduction unit depicted in Fig. 4 is one that decodes the signal transmitted from the access point and uses it to drive a set of logic gates to produce either a thermal or optical effect. The thermal or optical response stimulates the release of certain molecules encapsulated in a nanocarrier. In this sense, it is known that some nanocarriers such as liposomes can be designed in such a way that they release their content when stimulated by factors such as change in temperature, light intensity, $\mathrm{pH}$, magnetic field and enzymes [35]. In our design, we consider two sets of liposomes that respectively respond to temperature and light variations to release their contents. Each set of liposomes contains different molecules. Let the molecule type encapsulated by the thermal responsive liposome be $G_{E}$ and that encapsulated by the photo-responsive liposome be $G_{D}$. As is illustrated in Fig. 5(a), when the decoded command is the binary code 011 , the thermal source around the liposome storage unit is in the $\mathrm{ON}$ state. Hence, the release of $\mathrm{G}_{\mathrm{E}}$ occurs because of the thermal stimulation of the thermal responsive liposomes. When the decoded command is the binary code 111, the optical source around the liposome storage unit is in the $\mathrm{ON}$ state. Hence, the release of $\mathrm{G}_{\mathrm{D}}$ occurs owing to the optical stimulation of the photo-responsive liposomes to release their contents, as is illustrated in Fig. 5(b).

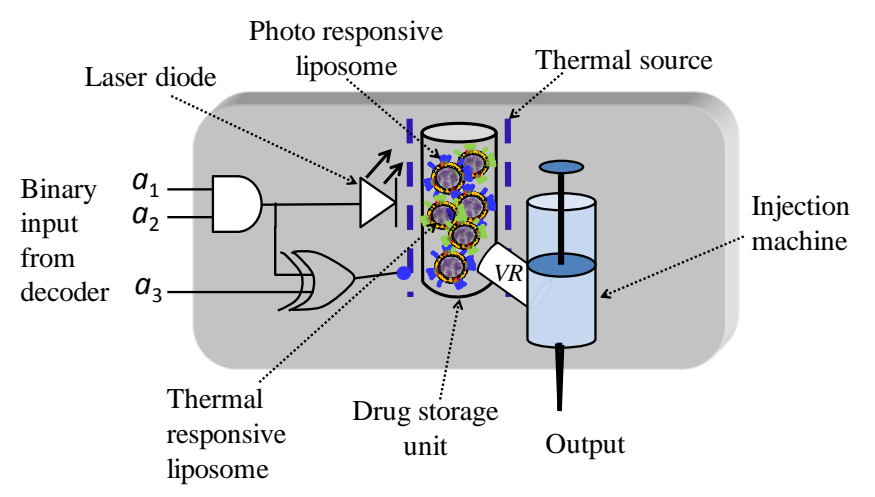

Fig. 4. Schematic diagram of the electro-bio transduction unit.

The above design is such that the released molecules diffuse through the space depicted by VR into the injection chamber, where they are automatically injected into the blood vessel network using a system that is capable of painless injection. It is shown in [36] that such painless injection system can be obtained by 
applying pressure, vibration, and cooling or warming to the skin. The injected molecules traverse through the blood network and passively enter the targeted site to excite the appropriate nanodevices.

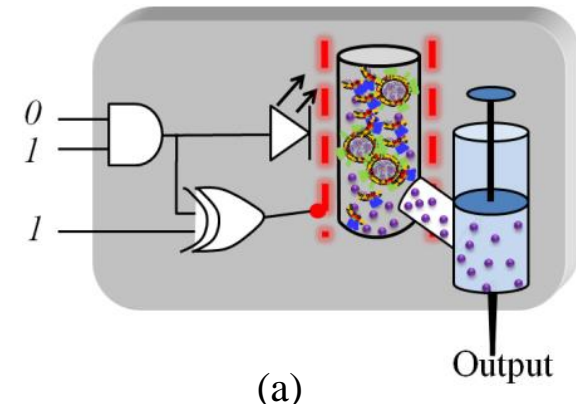

(a)

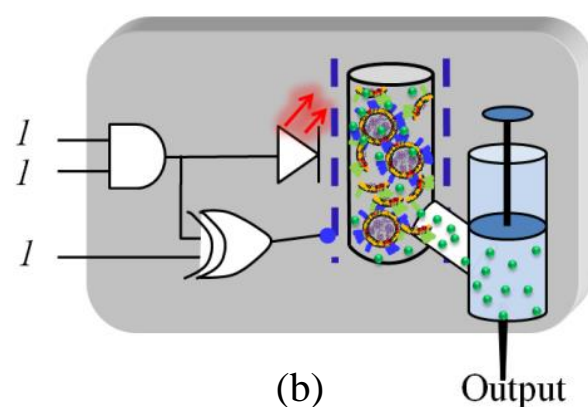

(b)

Fig. 5. Schematic diagram of (a) the thermo-triggered electro-bio transduction unit in ON state (b) the photo-triggered electro-bio transduction unit in $\mathrm{ON}$ state.

The release process of the molecules encapsulated by the liposomes can be expressed as [37]

$$
v(t)=\psi_{T}\left(1-e^{-\gamma t}\right)
$$

where $\gamma$ is the release rate equivalent to the first-order rate constant, and $\psi_{T}$ is the cumulative concentration of the released molecules. The release rates of the thermal responsive liposome and the photo-responsive liposome are $\gamma_{t}$ and $\gamma_{p}$, respectively, where $\gamma_{t}, \gamma_{l} \in \gamma$.

Since we are interested in the concentration released at a given time instant, we can express this concentration as the density function given by

$$
\psi(t)=\frac{d \psi_{T}\left(1-e^{-\gamma t}\right)}{d t}
$$

where $\psi_{T} \approx \int_{0}^{\infty} \psi(t) d t$.

In the case of photo-responsive liposomes, we consider release by photoisomerization, where the release of encapsulated content from liposomes is typically achieved by embedding a molecule that experiences a conformational change upon light illumination into the bilayer membrane. The conformational change destabilizes the lipid membrane and allows release.

On the other hand, thermo-responsiveness is usually governed by a nonlinear sharp change in the 
properties of at least one component of the nanocarrier material with temperature. Such a sharp response triggers the release of the drug following a variation in the surrounding temperature. Ideally, thermosensitive nanocarriers should retain their load at body temperature $\left(\sim 37^{\circ} \mathrm{C}\right)$ and rapidly deliver the drug within a locally heated microenvironment $\left(\sim 40-42^{\circ} \mathrm{C}\right)$.

The injection machine injects the released molecules at a time $t_{\mathrm{D}}$ after a period $T_{\mathrm{IN}}$ defined by

$$
T_{I N}=t_{D}-t_{R}
$$

where $t_{\mathrm{R}}$ is the time at which the release process starts. We express the output of the electro-bio unit as

$$
\begin{gathered}
c^{(f)}=\int_{0}^{T_{I N}} \xi \psi(t) d t \\
\psi_{0}=\left.\xi \psi(t)\right|_{t=T_{I N}}
\end{gathered}
$$

where $\xi$ is the total number of liposomes that released their content.

Let $v_{0}$ be the value of $c^{(f)}$ required at the nanonetwork site, which ensures that accurate information is delivered to $n T R_{2}$ and $n T R_{3}$. The target is to ensure that appropriate $\psi_{0}$ and period $T_{\mathrm{IN}}$ are employed in the IoBNT system in order to achieve the desired information molecule concentration $v_{0}$.

\section{B. Bio-electro interface model}

Now let us consider the reverse communication, that is, the link containing the bio-electro transduction unit. This unit mainly works on the principle of bioluminescence reaction. In this case, the bio-cyber interface detects the presence of the information molecules $G_{\mathrm{C}}$ in the blood vessel network and converts the information to an equivalent electric one. We model a conceptual bio-electro transduction unit as a subdermal/transdermal system with receptor-like probes/nanopores that extend into the blood vessel, as shown in Fig. 6. The model we present in this work comprises the sensor part and the transduction part. The sensor part consists of a synthesized or genetically modified cellular structure whose membrane receptors or nanopores act as probe into the vascular system. When the information molecules $G_{\mathrm{C}}$ that are released by the nanosensor enter the vascular system, they circulate and are detected by the receptor probes. Depending on the molecules to be detected, the probe can be complementary biochemical molecules such 
as monoclonal antibodies [38]. The detection process can be a ligand-receptor binding action between the circulating information molecule and the receptor probe. In this case the architecture shown in Fig. 7(a) suffices. On the other hand, the information molecules may directly diffuse into the cellular structure, in which case the architecture shown in Fig. 7(b) can be used. The cellular structure can be a bioluminescent bioreporter [39-42] that produces a reporter protein, which in this case is a luciferase (LU), on excitation by an analyte. The bioreporter can be developed by engineering LU genes genetically in other living cells [43]. Prototype circuits have been developed in recent times $[44,45]$. The analyte in this case is the information molecules emitted by the nanosensor and circulating in the vascular system. The synthesized LU molecules undergo a bioluminescence reaction with luciferin (L) in the bioreporter. The emitted light is sensed by a nano-scale highly sensitive light sensor, which in turn generates an equivalent output electric signal. The electric signal drives the transmitter to transmit the information-carrying wave through the wireless channel. For higher sensitivity, a photomultiplier can be used instead of the photoresistor shown in Fig. 7.

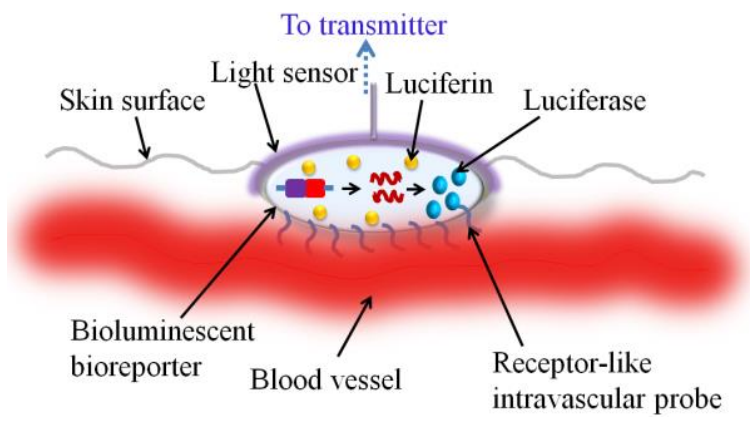

Fig. 6. Schematic of the bio-electro transduction unit.

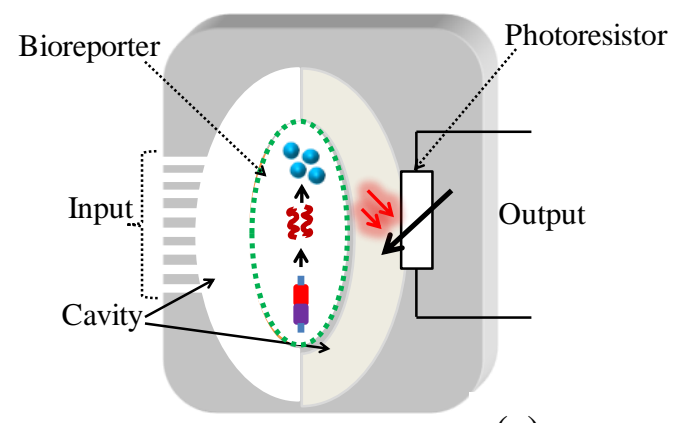

(a)

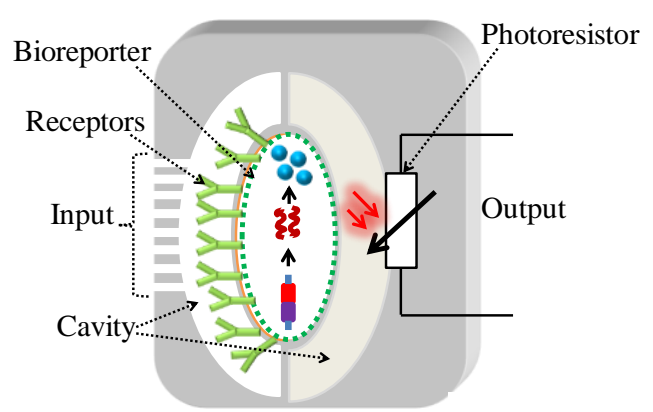

(b)

Fig. 7. Schematic of the bio-electro transduction unit for (a) direct diffusion scenario (b) receptor-mediated scenario. 
The bioluminescence reaction is a complex enzymatic reaction in which the LU catalyzes L oxidation by oxygen in the presence of adenosine triphosphate (ATP) and $\mathrm{Mg}^{2+}$, as shown below [46].

$$
\begin{aligned}
\mathrm{L}+\mathrm{O}_{2}+\mathrm{ATP} \stackrel{\text { Luciferace, } \mathrm{Mg}^{2+}}{\longrightarrow} & \text { Oxylucifer in }+\mathrm{CO}_{2} \\
& +\mathrm{AMP}+\mathrm{PP}+h v
\end{aligned}
$$

where adenosine monophosphate (AMP), phosphate group (PP), carbon dioxide, and light represented as $h v$ are the reaction products.

We shall assume that the concentration of the L and oxygen are much higher than that of LU and ATP, so that we can simplify the equation complexity and treat it as a simple one-substrate enzyme reaction kinetic involving the LU and ATP. Thus, following the Michaelis-Menten mechanism, the rate of producing light, which we simply refer to as bioluminescence intensity $I$, can approximately be expressed as

$$
I(t)=\frac{\alpha_{l} l_{a} a t p}{a t p+\alpha_{M}}
$$

where $l_{a}$ and atp are the concentrations of the LU and ATP, respectively. The terms $\alpha_{l}$ and $\alpha_{M}$ are the catalytic reaction constant and the Michaelis-Menten constant, respectively.

We can obtain the expression for $l_{a}$ by considering the following regulated gene expression differential equations [24]:

$$
\begin{aligned}
& \frac{d m_{R N A}}{d t}=\kappa_{r} g(B)-\gamma_{r} m_{R N A} \\
& \frac{d l_{a}}{d t}=\kappa_{p} m_{R N A}-\gamma_{p} l_{a}
\end{aligned}
$$

where $m_{R N A}$ is the messenger ribonucleic acid (mRNA), $g(B)$ is the transcription function regulated by the transcription factor $B$, and $\kappa_{r}=\left(\alpha_{0}-\alpha_{1}\right) P_{T}$ [24]. The terms $\kappa_{p}, \gamma_{p}$ and $\gamma_{r}$ are the rate constant of translating mRNA into LU, $\gamma_{p}$ is the degradation rate of LU, and $\gamma_{r}$ is the degradation rate of mRNA. The terms $\alpha_{0}$, $\alpha_{1}$ and $P_{T}$ are the basal mRNA production rate, the regulated mRNA production rate and the total amount of the free and bound promoter sites, respectively. The function $g(B)$ can generally be expressed as a Hill function [47], 
$g(B)=\frac{b(t)^{n}}{\theta^{n}+b(t)^{n}}$

where $b$ is the effective concentration of $B, \theta$ is the Hill constant that defines the knee of the rate of LU synthesis, and $n$ is a coefficient that defines its roll off. Assuming steady state, and for $n=2$ [48], then,

$l_{a}=\frac{\kappa_{r} \kappa_{p}}{\gamma_{p}}\left(\frac{b(t)^{2}}{\gamma_{r}\left(\theta^{2}+b(t)^{2}\right)}\right)$.

Typically, in natural cells, the transcription factors are a group of proteins found inside the cells. These proteins often exist in an inactive state until they are activated by the downstream activities of the cellular signaling pathways initiated by some extracellular signaling. Hence, the binding of the information molecules released by the nanosensor to the receptor-like intravascular probe or the direct diffusion of the molecules into the bioreporter excites the cellular signaling pathway, leading to the formation of certain molecules that bind to the inactive transcription factors to activate them. Usually, the regulation of genes by the transcription factor entails the following steps: polymerization and promoter binding [49]. For a single transcription factor, the rate of change of the concentration of $B$ is given by [24],

$$
\frac{d b}{d t}=k_{p 0} b-k_{p} b-\gamma_{B} b
$$

where $k_{p 0}, k_{p}$, and $\gamma_{B}$ are the reverse rate of polymerization, the forward rate of polymerization, and the decay rate of $B$, respectively.

In [24], the transcription factor was modeled as an oscillatory input. In this present work, we reasonably consider that the rate at which the transcription factor is activated through the pathway initiated by the receptor-mediated or direct diffusion of the information molecules into the cellular structure depends on the concentration of the diffusing information molecules. Hence, in relation to the biological signals $c^{(r)}(t)$ that go into the bio-cyber interface, we can express the concentration of the transcription factor as

$$
b(t) \approx \eta c^{(r)}(t)
$$

where $\eta$ accounts for the signal conditioning at the cellular structure surface through which the molecules diffuse. 
Let $I_{0}$ be the illumination intensity required to switch on the transmitter. Hence, when $I(t) \geq I_{0}$, the transmitter is on, but when $I(t) \leq I_{0}$ the transmitter is in the OFF state. The target is to ensure that for some $\alpha_{l}$ and $\alpha_{M}$, the appropriate concentration of LU and ATP are available to ensure that $I(t) \geq I_{0}$ is obtained.

\section{Compartmental Model for Molecule Propagation through the Blood Network}

We employ the compartment model [31] to model the concentrations of the propagating information molecules that are in the blood vessel network, and the ones that eventually reach the nanonetwork after being injected. A schematic overview of the compartment model is depicted in Fig. 8. In employing this model, the following assumptions are made. 1) The rate of nanoparticles' movement between compartments obeys a first-order reaction law, which is biophysically supported for a number of natural phenomena such as diffusion; 2) The system is a well-mixed one. This implies the instant homogeneous distribution of nanoparticles within the compartments; 3) The nanocarriers do not undergo chemical reactions on their course to the targeted sites; 4) The volumes of the compartments do not vary with time;

5) The drug is completely eliminated from the body through the blood compartment.

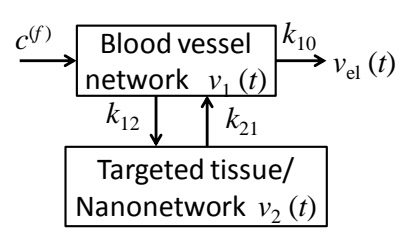

(a)

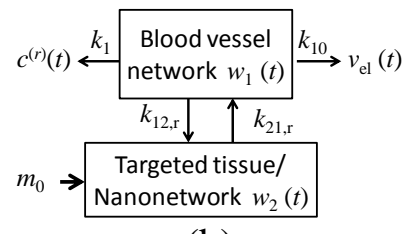

(b)

Fig. 8. Mammillary two-compartment model of in-body molecular propagation for (a) bio-cyber interface to nanonetwork, and (b) nanonetwork to bio-cyber interface.

The concentration of the information molecules in each compartment is given by the ratio of the number of molecules to the volume of the compartment. In Fig. 8(a) and (b), the central compartment is the blood system with the concentration of molecules designated as $v_{1}(t)$ and $w_{1}(t)$, respectively, while the concentration of molecules in the in-body nanonetwork of interest is designated as $v_{2}(t)$ and $w_{2}(t)$, respectively. The function $v_{\mathrm{el}}(t)$ is the concentration of eliminated or biochemically modified molecules over time as a function of the elimination rate $k_{10}$. This concentration includes molecules that undergo 
phagocytosis, reaction process, adhesion process, absorbed through non-targeted tissues, and eliminated by the liver. The parameters $k_{12} / k_{12, r}$ and $k_{21} / k_{21, r}$ are the first-order rate constant in or out of the nanonetwork compartment, respectively. These rate constants are typically dependent on the concentration difference between the compartments, the size of the fenestra through the endothelia cell network, and the properties of the diffusing information molecules [50]. The following rate equations arise from Fig. 8(a).

$$
\begin{aligned}
& \frac{d v_{1}(t)}{d t}=-v_{1}(t)\left(k_{12}+k_{10}\right)+k_{21} v_{2}(t) \\
& \frac{d v_{2}(t)}{d t}=k_{12} v_{1}(t)-k_{21} v_{2}(t)
\end{aligned}
$$

with the initial conditions $v_{1}(0)=c^{(f)}$, and $v_{2}(0)=0$. For Fig.8 (b), we consider the reverse of the conventional two-compartment model and express rate equations as

$$
\begin{aligned}
& c^{(r)}(t)=k_{1} w_{1}(t) \\
& \frac{d w_{1}(t)}{d t}=k_{21, r} w_{2}(t)-\left(k_{12, r}+k_{10}+k_{1}\right) w_{1}(t) \\
& \frac{d w_{2}(t)}{d t}=k_{12, r} w_{1}(t)-k_{21, r} w_{2}(t)
\end{aligned}
$$

with the initial conditions $w_{1}(0)=0$ and $w_{2}(0)=m_{0}$. The term $m_{0}$ represents the total concentration of the molecules released by the nanosensor, assuming an instantaneous release of molecules by the nanosensor. The kinetic constants $k_{12, r}$ and $k_{21, r}$ are the reverse equivalents of $k_{12}$ and $k_{21}$. We associate the parameter $k_{1}$ with the dissociation constant of the ligand-receptor binding in the case of the receptor-like probe or membrane diffusion constant in the case of direct diffusion of information molecules into the bio-electro induction unit. Typically, the movement of molecules in and out of the compartments and through the blood network is based on Brownian motion. Hence, we model the molecular concentrations as containing Gaussian noise [51]. Thus

$$
v_{i}(t), w_{i}(t) \sim \mathcal{N}\left(0, \sigma^{2}\right)
$$

where $\mathcal{N}\left(0, \sigma^{2}\right)$ is a zero-mean process with variance, $\sigma^{2}$. 


\section{Channel Capacity of IoBNT}

A capacity expression of the diffusion-based MC system is given in [52] and that of the cardiovascular system is given in [53] with respect to the TDD scenario. These expressions discretely represents the different links in the entire IoBNT, where [52] defines the capacity between a nanodevice and another within the nanonetwork, and [53] defines information capacity between the nanonetwork and the bio-cyber interface. To obtain a unified capacity expression for the entire link from the access point, AP to the nanonetwork NX, we can apply the degraded relay channel [54] abstraction to represent the system as shown in Fig. 9.

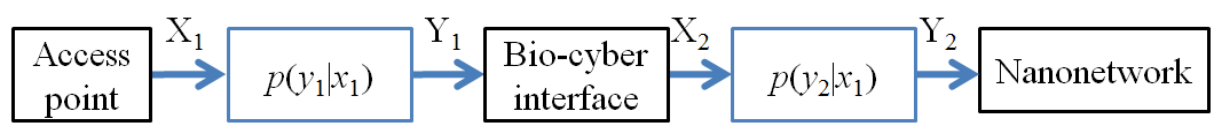

Fig. 9. Degraded relay channel equivalent of the IoBNT.

The interpretation is the bio-cyber interfaces acts as a relay in the system. In this pursuit, $x_{1}$ is the input to the wireless channel specified by $p\left(y_{1} \mid x_{1}\right)$ and $y_{1}$ is the output, $x_{2}$ is the relay's output and $y$ is the output of the cardiovascular system specified by $p\left(y_{2} \mid x_{2}\right)$ as shown in Fig. 9. Hence, we can the joint probability as $p\left(y_{1}, y_{2} \mid x_{1}\right)=p\left(y_{1} \mid x_{1}\right) p\left(y_{2} \mid y_{1}\right)$. For the degraded relay channel under the consideration, assuming that the knowledge of $y_{1}$ invariably gives $x_{2}$, then, $p\left(y_{2} \mid x_{1}, x_{2}, y_{1}\right)=p\left(y_{2} \mid x_{2}, y_{1}\right)$. Therefore, following [54], we can express the capacity $C$ of the IoBNT channel in Fig. 9 as

$$
C=\sup _{p\left(x_{1}, x_{2}\right)} \min \left\{I\left(X_{1}, X_{2} ; Y_{2}\right), I\left(X_{1} ; Y_{1} \mid X_{2}\right)\right\}
$$

where the supremum is over all joint probability $p\left(x_{1}, x_{2}\right)$.

\section{Simulation ResUlts AND DisCUSSION}

In this section, we provide the quantitative simulation results and performance evaluation of the IoBNT. The analytical expressions given in Section III and IV are evaluated. This evaluation focuses on the proposed bio-cyber interface and the blood network complex, as indicated by the red lines in Fig. 10. Specifically, given that on the release of the first drug $G_{A}$, the nanosensor detects $G_{A}$ and releases $G_{B}$ of 
total concentration $m_{0}$, we follow the different transition states of the $m_{0}$ though the IoBNT network until $I(t)$, which translates to $a^{(r)}$, where the value of $a^{(r)}$ is either 1 (transmitter ON) for $I(t) \geq I_{0}$ or 0 (transmitter $\mathrm{OFF}$ ) for $I(t) \leq I_{0}$. The effects of some critical system and design parameters such as $m_{0}, k_{21, r}, k_{1}, k_{10}, \alpha_{\mathrm{M}}$ and the concentration of ATP on $a^{(r)}$ are evaluated. Given that the medical personnel sends out the command binary code 011 or 111 to $n T R_{2}$ or $n T R_{3}$ in the in-body nanonetwork through the Internet to start the delivery of drugs, we also follow the different transition states of the properly received and decoded sequence $a^{(f)}$ as it goes though the IoBNT network until it is received as a drug concentration $v_{2}(t)$. The effects of critical design parameters such as $\psi_{0}, \gamma, T_{I N}$, and $k_{12}$ on the output $v_{2}(t)$ are also evaluated.

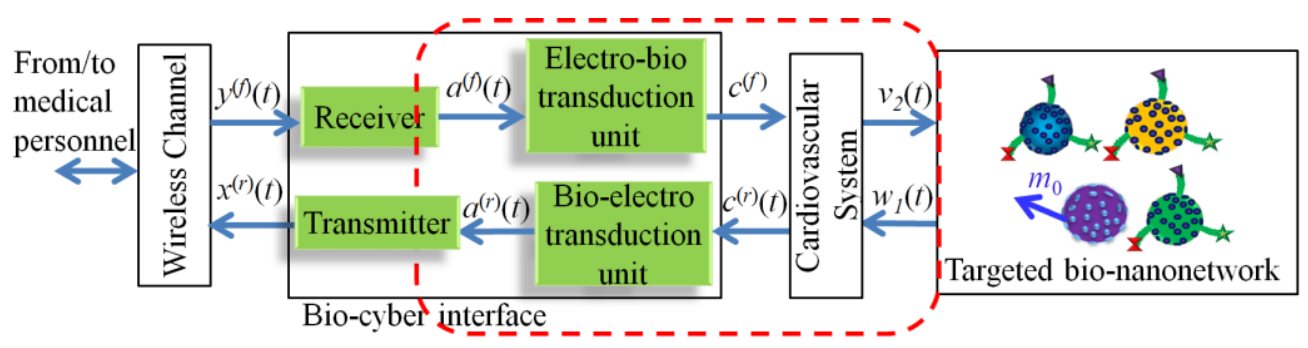

Fig. 10. Diagram of the evaluated bio-cyber interface-blood network complex.

In the simulation, the values of the evaluation parameters used are taken from the literature and scaled up or down in the same order. The values of the compartment model parameters used are $k_{21}=0.00103 \mathrm{~min}^{-1}$, $k_{12}=0.373 \mathrm{~min}^{-1}$ and $k_{10}=0.172 \min ^{-1}[55] ; k_{12, r}=0.00103 \mathrm{~min}^{-1}, k_{21, r}=0.373 \mathrm{~min}^{-1} ;$ and $k_{1}=0.001 \mathrm{~min}^{-1}$. We set $\eta=1$ by assuming direct diffusion of information molecules into the bio-electro induction unit. The parameters for the expression of LU are as follows: $\kappa_{r}=10$ [56], $\kappa_{p}=150 \mathrm{~h}^{-1}$ [57], $\gamma_{r}=10.05 \mathrm{~h}^{-1}$ [58], and $\gamma_{p}=4.15 \mathrm{~h}^{-1}[58]$. For the bioluminescence reaction, the values of the parameters used are, $\alpha_{\mathrm{M}}=15 \mu \mathrm{M}, \alpha_{l}$ $=4.4 \times 10^{-2}$ and $a t p=40 \mu \mathrm{L}$ [59]. The noise variance used in the simulation is $0.05 \mu \mathrm{M}$ [60]. In analyzing the bioluminescence intensity, we assume that the wavelength of the emitted light is approximately constant, which is reasonable if the emitted light color does not vary significantly. Hence, the sensitivity of the photoresistor will basically depend on the intensity of the emitted light. By using the experimental data in [61] for liposome exposure to ultraviolet light, the value of $\gamma_{l}=0.000104 \mathrm{~min}^{-1}$ is adopted in our work. 
In this work, we use $\gamma_{t}=0.0078 \mathrm{~min}^{-1}$ [62] obtained by using the best fitting curve on nonlinear least square methods for experimental data obtained at $42^{\circ} \mathrm{C}[63]$.

For the communication between the nanosensor and the medical personnel, the effects of varying values of $m_{0}, k_{21, r}, k_{1}, \alpha_{\mathrm{M}}$, atp and $k_{10}$ on the bioluminescence intensity $I(t)$ expressed in arbitrary unit (a.u) are shown in Fig. 11. The results are obtained from (7), (11), (13), (16) and (17). Let us assume that $I_{0}$ is equal to 0.7 a.u on the normalized scale. In Fig. 11(a), it can be observed that a low value of $m_{0}$, say less that 80 $\mu \mathrm{M}$, will undesirably be unable to turn on the transmitter. Hence, the nanosensor should be capable of releasing a high concentration of information molecules to ensure that it is efficiently received at the biocyber interface. This can be achieved by using multiple nanosensors to increase overall capacity. In Fig. 11(b), the effect of variation in the forward rate constant $k_{21, r}$ on the bioluminescence intensity is shown. As stated earlier, this parameter typically depends on the concentration difference between compartment 2 and 1 , the size of the fenestra through the endothelia cell network that separates the nanonetwork from the blood network, and the properties of the diffusing information molecules. It is shown in Fig.11 (b) that as $k_{21, r}$ increases, more molecules are received by the bio-cyber interface, resulting in higher bioluminescence.

In Fig. 11(c), it is shown that the bioluminescence intensity significantly increases with the rate at which the information molecules are detected by the bio-cyber interface. This rate depends on the concentration of the receptor-like probe or the number, size (in relation to the size of the information molecule) and distribution of the membrane pores through which molecules enter the bio-cyber interface. In Fig. 11(d), we show the effect of variations in the bioluminescence Michaelis-Menten reaction constant $\alpha_{\mathrm{M}}$ on the light intensity. It can be observed that within the known range of 10-17 $\mu \mathrm{M}$ for firefly [59], there is little effect of this constant on the bioluminescence intensity compared to other parameters. The effect of the concentration of ATP on the bioluminescence intensity is also shown in Fig. 11(e), where the trend indicates that an increase in atp results in higher bioluminescence intensity.

Typically, we do not have much control over the rate parameter $k_{21, r}$ since it is greatly dependent on the in-body system. However, we have control over the design parameters such as $m_{0}, k_{1}, a_{\mathrm{M}}$ and atp. Hence, 
given a low $k_{21, r}$, it is desired to find a set of parameters with minimal elements whose values can be adjusted to ensure efficient communication. Of the following parameters $k_{1}, a_{\mathrm{M}}$ and atp, it can be observed from Fig. 11(f) that $k_{1}$ has a significant effect on the bioluminescence intensity. Hence, given some $m_{0}$ and low $k_{21, r}$ we can increase the reliability of transmission by increasing $k_{1}$. We can achieve this by ensuring that the bio-electro transduction unit surface exposed for the detection of the information molecule is large enough. This can be achieved by increasing the density of the probes or the nanopores through which

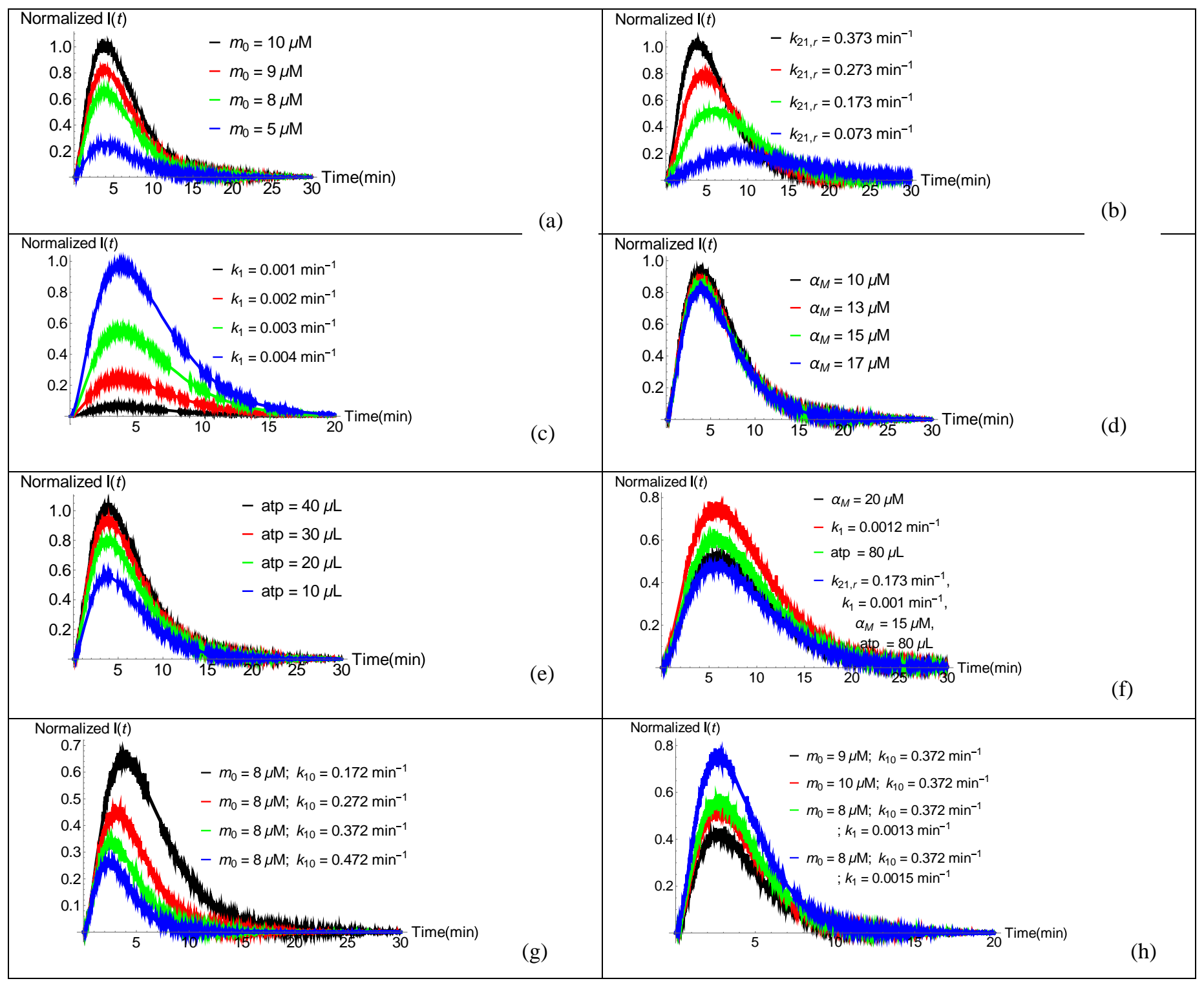

Fig. 11. Variation in the bioluminescence intensity with (a) concentration of $m_{0}$ (b) the diffusion rate constant $k_{21, r}(\mathrm{c}) k_{1}(\mathrm{~d})$ the Michaelis-Menten constant $\alpha_{\mathrm{M}}(\mathrm{e})$ the concentration of atp (f) with concentration of $\alpha_{\mathrm{M}}, k_{1}$, atp, and $k_{21, r}(\mathrm{~g})$ constant $m_{0}$ and varying $k_{10}(\mathrm{~h})$ constant $k_{10}$ and varying $m_{0}$ and $k_{1}$. 
molecules diffuse into the cellular structure/bioreporter. In addition, bioluminescence intensity can be increased further by using genetic modification that combines various mutations in LU [64]. Another parameter of great importance is the $k_{10}$, which accounts for the loss of the information molecules by absorption through non-targeted tissues, phagocytosis, reaction process, adhesion process elimination by liver. It can be seen in Fig. 11(g) that this parameter greatly influences the performance of the IoBNT. The degradation in performance caused by increase in $k_{10}$ can be ameliorated by increasing the $m_{0}$ and/or $k_{1}$. In Fig. 11(h) it can be seen that a slight increase (less than $0.0005 \mathrm{~m}^{-1}$ ) in $k_{1}$ raises the bioluminescent level significantly compared to a $1 \mu \mathrm{M}$ increase in $m_{0}$. Hence, $k_{1}$ is a crucial parameter for improving IoBNT performance.

Next, we consider the communication between the medical personnel and the nanosensor through the electro-bio transduction unit. The effects of varying the values of parameters such as $\psi_{0}, \gamma, T_{I N}$, and $k_{12}$ on the concentration of information molecules $v_{2}(t)$ that enter the nanonetwork site are shown in Fig. 12. The results are obtained from (3), (4), (5), (14) and (15). In Fig. 12(a), the effects of different values of the concentration of the message molecules $\psi_{0}$, which are encapsulated by the liposomes in the electro-bio transduction unit, on $v_{2}(t)$ are shown. It can be seen that as expected, $v_{2}(t)$ increases with higher values of $\psi_{0}$. Let us assume that $v_{0}$, which is the concentration of molecules that are required at the nanonetwork site to ensure that accurate information is delivered to $n T R_{2}$ and $n T R_{3}$, has the normalized value of 0.7 . With $\lambda=0.0 .104 \mathrm{~min}^{-1}$ and $T_{\mathrm{IN}}=30 \mathrm{~min}$, it can be inferred from Fig. 12(a) that in this simulation, a concentration of $\psi_{0}$ less than $0.7 \mathrm{~mL}$ will be inadequate for efficient communication. The portion of $\psi_{0}$ injected, which is $c^{(f)}$, is controlled by the release rate of the liposome $\lambda$ and the period $T_{\mathrm{IN}}$ between the commencement of molecule release and injection time, as shown in Fig. 12(b) and (c), respectively. It can be seen from Fig. 12(b) and (c) that an increase in respectively $\lambda$ and $T_{\mathrm{IN}}$ results in an increase in $v_{2}(t)$. The release rate $\lambda$ depends on the type of stimuli that trigger the release and the liposome structure and composition. For instance, based on the $\lambda$ of the photo-responsive and the thermo-responsive liposome previously stated, it is obvious that the $c^{(f)}$ of the latter will be greater than that of the former, for the same 
$\psi_{0}$ and $T_{\mathrm{IN}}$

The effect of the forward rate constant $k_{21}$ on $v_{2}(t)$ is shown in Fig. 12(d), where it can be seen that an increase in $k_{21}$ results in a higher value of $v_{2}(t)$. In relation to our previous discussion, this rate depends on the concentration difference between the compartments, the size of the fenestra through the endothelia cell network that separates the nanonetwork and the blood network, and the properties of the diffusing information molecules. Since we do not have much control over this parameter, it is imperative to ensure that $\lambda$ and $T_{\mathrm{IN}}$ are high enough to give high $c^{(f)}$, and subsequently $v_{0}$. While the effect of $k_{10}$ is not shown in Fig. 12, it can be inferred from our earlier assertion that $k_{10}$ greatly influences the performance of the IoBNT, that significantly influences $v_{2}(t)$.

Finally, it is important to note that the above analysis is unique to the bio-cyber interface architecture presented in this work. A different architecture and mechanism of the bio-cyber interface will definitely present different parameters, requirements and analysis. Note also that aside from the compartmental model used to represent the propagation of the molecules through the blood network; models such as the ones presented in $[8,65]$ can provide more information and better accuracy. Moreover, the inclusion of errors that arise from the Internet connection will give a more realistic system performance analysis.

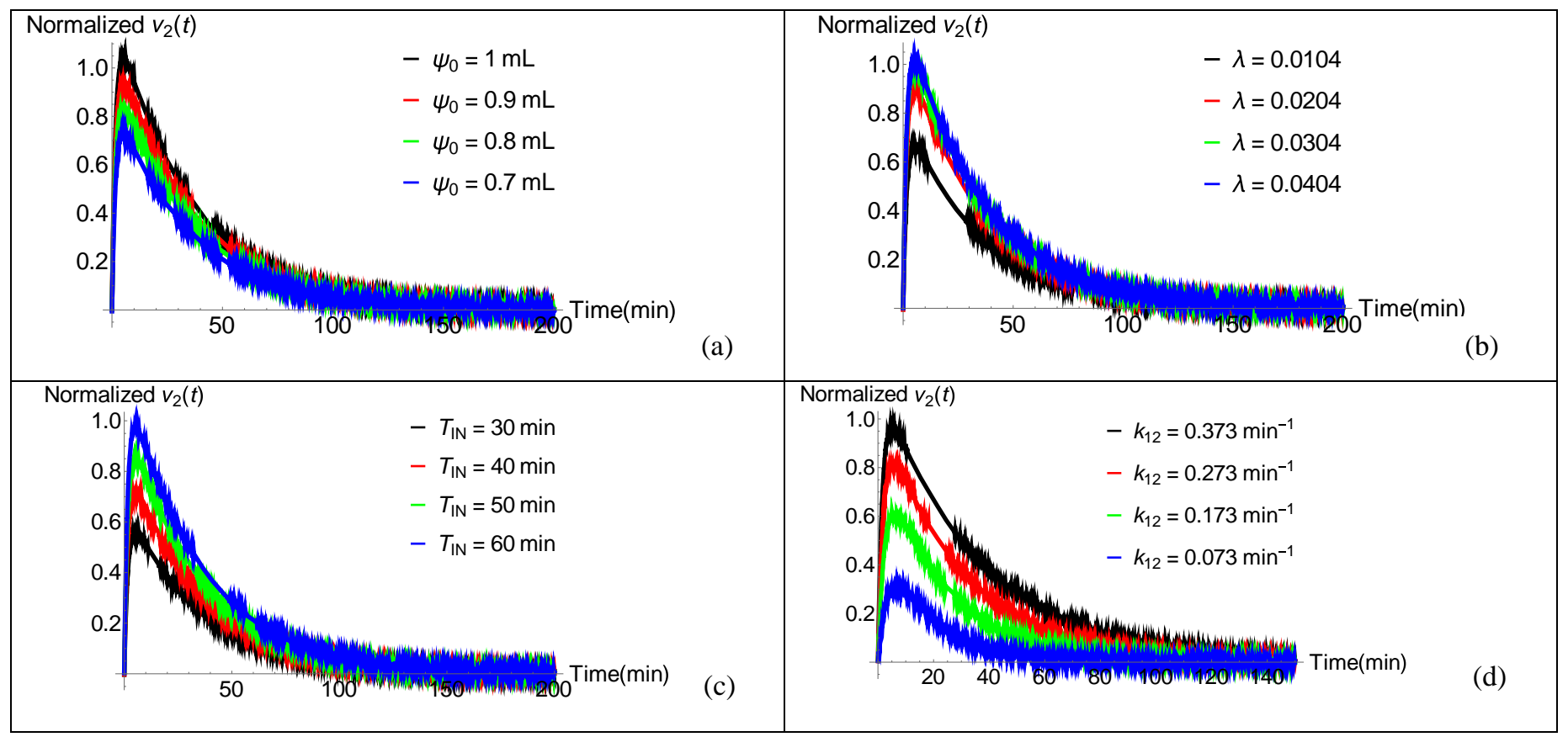

Fig. 12. Variation in $v_{2}(t)$ delivered to the in-body nanonetwork, with (a) $\psi_{0}$ (b) $\lambda$ (c) $T_{\mathrm{IN}}$. (d) $k_{12}$. 


\section{CONCLUSION}

In this paper, we have provided an illustration and model example of the IoBNT that is based on a prospective realistic scenario in advanced healthcare delivery. We have also presented a design example for the bio-cyber interface, which is an important unit in the overall IoBNT. The proposed architecture of the bio-cyber interface employed the concept of thermal and light responsiveness of biochemical molecules for transduction of an electrical signal to a biochemical signal and the phenomenon of bioluminescence reaction for the transduction of a biochemical signal to electrical signals. The propagation of the biochemical signal through the body is achieved through the blood vessel medium. To model this propagation, we employed the two-compartment pharmacokinetic model. The parameters associated with the compartment model were referred to as system parameters, which are intrinsic to the patient's body system, while the parameters associated with the bio-cyber interface were termed design parameters. The effects of the system and design parameters on the IoBNT performance were analyzed. The analysis was based on numerical results from simulations. It was shown that while we do not necessarily have control over the system parameters, the choice of the design parameters can go a long way in improving system performance and overcoming the challenges posed by the system parameters.

\section{ACKNOWLEDGMENT}

This research work was funded by the Sentech Chair in Broadband Wireless Multimedia Communications at the University of Pretoria and the Department of Trade and Industry THRIP Program.

\section{REFERENCES}

[1] I. Akyildiz, F. Brunetti, and C. Blázquez, "Nanonetworks: A new communication paradigm," Computer Networks, vol. 52, no. 12, pp. 2260-2279, 2008.

[2] M. Pierobon, "A systems-theoretic model of a biological circuit for molecular communication in nanonetworks," Nano Communication Networks, vol. 5, pp. 25-34, 2014. 
[3] S. Balasubramaniam, and P. Lio, "Multi-hop conjugation based bacteria nanonetworks," IEEE Trans. NanoBio., vol. 12, no. 1, pp. 47-59, 2013.

[4] T. Nakano, T. Suda, Y. Okaie, M. Moore, and A. Vasilakos, "Molecular communication among biological nanomachines: A layered architecture and research issues," IEEE Trans. NanoBio., vol. 13, no. 3, pp. 169-197, 2014.

[5] A. Noel, K.C. Cheung, and R. Schober, "Improving receiver performance of diffusive molecular communication with enzymes," IEEE Trans. NanoBio, vol. 13, no. 1, pp. 31-43, 2014.

[6] Y. Chahibi, M. Pierobon, S.O. Song, and I.F. Akyildiz, "A molecular communication system model for particulate drug delivery systems," IEEE Trans. Biomed Eng, vol. 60, no. 12, pp. 3468-3483, 2013.

[7] U.A.K. Chude-Okonkwo, "Diffusion-controlled enzyme-catalyzed molecular communication systems for targeted drug delivery," IEEE Global Communication Conference, Austin, Texas, Dec. 8-12, 2014.

[8] Y. Chahibi, M. Pierobon, and I. Akyildiz, "Pharmacokinetic modeling and biodistribution estimation through the molecular communication paradigm," IEEE Trans. Biomed. Eng., vol. 62, no. 10, pp. 2410$2420,2015$.

[9] B. Atakan, O.B. Akan, and S. Balasubramaniam. "Body area nanonetworks with molecular communications in nanomedicine." IEEE Commun. Mag., vol. 50, no. 1, pp. 28-34, 2012.

[10] L. Felicettia, M. Femminellaa, G. Realia, and P. Liòb, " Applications of molecular communications to medicine: A survey," Nano Commun. Netw., 2010. doi:10.1016/j.nancom.2015.08.004

[11] A.E. Nel et al, " Understanding biophysicochemical interactions at the nano-bio interface," Nat. Mater, vol. 8, no. 7, pp. 543-557, 2009.

[12] T. Nakano, M. Moore, Y. Okaie, A. Enomoto, and T. Suda, "Swarming biological nanomachines through molecular communication for targeted drug delivery," in SCIS-ISIS 2012, 2012.

[13] T. Nakano, T. Suda, Y. Okaie, M. J. Moore, and A. V. Vasilakos, "Molecular communication among biological nanomachines: A layered architecture and research issues," IEEE Trans. NanoBio., vol. 13 , no. 3, pp. 169-197, 2014. 
[14] I. F. Akyildiz, M. Pierobon, S. Balasubramaniam, and Y. Koucheryavy. "The Internet of Bio-Nano things," IEEE Commun. Mag., vol. 53, no. 3, pp. 32-40, 2015.

[15] U.A.K. Chude-Okonkwo, R. Malekian, and B.T. Maharaj, "Bio-inspired approach for eliminating redundant nanodevices in Internet of Bio-NanoThings," in IEEE Globecom Workshops (GC Wkshps), 6-10 Dec. 2015, San Diego, USA.

[16] L. Ma, M. Kohli, and A. Smith, "Nanoparticles for combination drug therapy," ACS nano, vol. 7, no. 11 , pp. 9518-9525, 2013.

[17] J. Woodcock, J.P. Griffin, and R.E. Behrman, "Development of novel combination therapies," New England Journal of Medicine, vol. 364, no. 11, pp. 985-987, 2011.

[18] A. I. Elegbede et al., "Mechanistic studies of the triggered release of liposomal contents by matrix metalloproteinase-9," J. of the American Chemical Society, vol. 130, no. 32, pp. 10633-10642, 2008.

[19] U.A.K. Chude-Okonkwo, R. Malekian, and B.T. Maharaj, "Molecular Communication Model for Targeted Drug Delivery in Multiple Disease Sites with Diversely Expressed Enzymes," IEEE Trans. Nanobio., to be published.

[20] P. P. Deshpande, S. Biswas, and V. P. Torchilin, "Current trends in the use of liposomes for tumor targeting," Nanomedicine, vol. 8, no. 9, pp. 1509-1528, 2013.

[21] V. P. Torchilin, "Multifunctional, stimuli-sensitive nanoparticulate systems for drug delivery," Nature Reviews Drug Discovery, vol. 13, no. 11, pp. 813-827, 2014.

[22] S. Basu, Y. Gerchman, C. H. Collins, F. H. Arnold, and R. Weiss, "A synthetic multicellular system for programmed pattern formation," Nature, vol. 434, no. 7037, pp. 1130-1134, 2005.

[23] C. J. Anderson, E. J. Clarke, A. P. Arkin, and C. A. Voigt, "Environmentally controlled invasion of cancer cells by engineered bacteria," J of Molecular Biology, vol. 355, no. 4, pp. 619-627, 2006.

[24] R. F. Fakhrullin, A. I. Zamaleeva, R. T. Minullina, S. A. Konnova, and V. N. Paunov, "Cyborg cells: functionalisation of living cells with polymers and nanomaterials," Chemical Society Reviews, vol. 41, no. 11, pp. 4189-4206, 2012. 
[25] B.D. Unluturk, A.O. Bicen, and I. F. Akyildiz, "Genetically engineered bacteria-based bioTransceivers for molecular communication," IEEE Trans. Commun., vol. 63, no. 4, pp. 1271-1281, 2015.

[26] U.A.K. Chude-Okonkwo, R. Malekian, B.T. Maharaj, and C.C. Olisah, "Bio-inspired physical layered device architectures for diffusion-based molecular communication: Design issues and suggestions," in 13th IEEE Intl. Conf. Ind. Info., 2015, Cambridge, 22-24 July 2015, pp.1472-1479.

[27] M.A. Dollard, and P. Billard, "Whole-cell bacterial sensors for the monitoring of phosphate bioavailability," Journal of Microbiological Methods 55, no. 1, pp. 221-229, 2003.

[28] D. Yeo, C. Wiraja1, Y. J. Chuah, Y. Gao, and C. Xu, "A nanoparticle-based sensor platform for cell tracking and status/function assessment," Scientific Reports, vol. 5, no. 14768, pp.1-14, 2015.

[29] M. A. Eckert, P. Q Vu, K. Zhang, D. Kang, M. M. Ali, C. Xu, C. and W. Zhao, "Novel molecular and nanosensors for in vivo sensing," Theranostics, vol. 3, no. 8, pp. 583-593, 2013.

[30] E. Monson, M. Brasuel, M. Philbert, and R. Kopelman. "PEBBLE nanosensors for in vitro bioanalysis," Biomedical Photonics Handbook 9, 2003.

[31] S. Dhillon, Clinical pharmacokinetics, Pharmaceutical Press, Graylake USA, 2006.

[32] C. R. Yonzon, D. A. Stuart, X. Zhang, A. D. McFarland, C. L. Haynes, and R. P. van Duyne, "Towards advanced chemical and biological nanosensors-an overview," Talanta, vol. 67, no. 3, pp. 438-448, 2005.

[33] L. Felicetti, M. Femminella, G. Reali, and P. Liò, "A molecular communication system in blood vessels for tumor detection," In Proceedings of ACM The First Annual International Conference on Nanoscale Computing and Communication, pp. 1-9, 2014.

[34] T. Nakano, S. Kobayashi, T. Suda, Y. Okaie, Y. Hiraoka, and T. Haraguchi, "Externally controllable molecular communication," IEEE J. Sel. Areas Commu., vol. 32, no. 12, pp..2417-2431, 2014. 
Q. Hu, P. S. Kattic, and Z. Gu, "Enzyme-responsive nanomaterials for controlled drug delivery," Nanoscale, vol. 6, no. 21, pp. 12273-12286, 2014.

[36] W.P. McKay et al., "Development of a painless injection device applying pressure, vibration, and temperature," 36th Annual Scientific Meeting of the Canadian Pain Society, May 20-23, 2015, Charlottetown, Canada.

[37] M. Afadzi, C. Davies and Y.H. Hansen, "Ultrasound stimulated release of liposomal calcein," in IEEE Conference on Ultrasonics Symposium (IUS), October 2010, pp. 11-14.

[38] A. Sukhanova, K. Even-Desrumeaux, P. Chames, D. Baty, M. Artemyev, V. Oleinikov and I. Nabiev, "Engineering of ultra-small diagnostic nanoprobes through oriented conjugation of singledomain antibodies and quantum dots," Nature Protocols/Protocols Exchange, 2012.

[39] D.M. Closea, T. Xua, A.E. Smartta, P. Jegiera, S. A. Rippa, and G.S. Sayler, "Light without substrate amendment: The bacterial luciferase gene cassette as a mammalian bioreporter," In SPIE Defense, Security, and Sensing, Intern. Society for Optics and Photonics, pp. 80290F-80290F, 2011.

[40] R. Vijayaraghavan et al., "A bioreporter bioluminescent integrated circuit for very low-level chemical sensing in both gas and liquid environments," Sensors and Actuators B: Chemical, vol. 123, no. 2, pp. 922-928, 2007.

[41] D. M. Close, S. Ripp, and G. S. Sayler, "Reporter proteins in whole-cell optical bioreporter detection systems, biosensor integrations, and biosensing applications," Sensors, vol. 9, no. 11, pp. 9147-9174, 2009.

[42] D.E. Nivens, T. E. McKnight, S. A. Moser, S.J. Osbourn, M. L. Simpson, and G.S. Sayler, "Bioluminescent bioreporter integrated circuits: potentially small, rugged and inexpensive whole - cell biosensors for remote environmental monitoring," J. Appl. Microbio., vol. 96, no. 1, pp. 33-46, 2004.

[43] C. H. Contag, and M. H. Bachmann, "Advances in in vivo bioluminescence imaging of gene expression," Annual Review of Biomedical Engineering, vol. 4, no. 1, pp. 235-260, 2002. 
[44] S. Rip et al., "Bioluminescent bioreporter integrated circuit (BBIC) sensors," In IEEE Bio Micro and Nanosystems Conference, 2006. BMN'06, pp. 59-60. 2006.

[45] E. K. Bolton et al., "Integrated CMOS photodetectors and signal processing for very low-level chemical sensing with the bioluminescent bioreporter integrated circuit," Sensors and Actuators B: Chemical, vol. 85, no. 1, pp. 179-185, 2002.

[46] S. M. Marques, and J. C. G. Esteves da Silva, "Firefly bioluminescence: A mechanistic approach of luciferase catalyzed reactions," IUBMB Life, vol. 61, no. 1, pp. 6-17, 2009.

[47] P. S. Gutierrez, D. Monteoliva, and L. Diambra, "Cooperative binding of transcription factors promotes bimodal gene expression response," PloS One, vol. 7, no. 9, Art. ID. e44812, 2012.

[48] G. Yagil, and E. Yagil, "On the relation between effector concentration and the rate of induced enzyme synthesis," Biophysical Journal, vol. 11, no. 1, pp. 11-27, 1971.

[49] R. Weiss et al., "Genetic circuit building blocks for cellular computation, communications, and signal processing," Natural Computing, vol. 2, no. 1, pp. 47-84, 2003.

[50] B. G. Klein, Cunnigham's Textbook of Veterinary Physiology, Missouri, USA: Elsevier Saunders, 2013, pp. 226.

[51] D. Z. d'Argenio, ed., Advanced methods of pharmacokinetic and pharmacodynamic systems analysis. Kluwer Academic Publishers, New York, 2004, pp. 107.

[52] M. Pierobon, and I. F. Akyildiz, "Capacity of a diffusion-based molecular communication system with channel memory and molecular noise,"IEEE Trans. Inf. Theory., vol. 59, no. 2, pp. 942-954, 2013.

[53] Y. Chahibi, and I. F. Akyildiz, "Molecular communication noise and capacity for particulate drug delivery systems," IEEE Trans. Commun., vol. 62, no. 11, pp. 3891-3903, 2014.

[54] A. M. Cover and A. A. El Gamal, "Capacity theorems for the relay channel," IEEE Trans. Inf. Theory, vol. IT-25, pp. 572-584, 1979.

[55] S. Cascone, G. Lamberti, G. Titomanlio, and O. Piazza, "Pharmacokinetics of Remifentanil: a threecompartmental modeling approach," Translational medicine@ UniSa, vol. 7, no. 4, pp. 18-22, 2013. 
H. Zhang, Y. Chen, and Y. Chen, "Noise propagation in gene regulation networks involving interlinked positive and negative feedback loops," PloS one, vol. 7, no. 12, Art. ID. e51840, 2012.

[57] C. M. Austin et al., "Modeling and validation of autoinducer-mediated bacterial gene expression in microfluidic environments," Biomicrofluidics, vol. 8, no. 3, Art. ID. 034116, 2014.

[58] T.S. Moon, C. Lou, A. Tamsir, B. C. Stanton, and C.A. Voigt, "Genetic programs constructed from layered logic gates in single cells," Nature, vol. 491, no. 7423, pp. 249-253, 2012.

[59] K. Niwa et al., "Quantum yields and kinetics of the firefly bioluminescence reaction of beetle luciferases," Photochemistry and Photobiology, vol. 86, no. 5, pp. 1046-1049, 2010.

[60] A.O Bicen, and I.F. Akyildiz, "End-to-end propagation noise and memory analysis for molecular communication over microfluidic channels," IEEE Trans. Commun., vol. 62, no. 7, pp. 2432-2443, 2014.

[61] N.K, Sharma, and V. Kumar, "Release kinetics of novel photosensitive liposome for triggered delivery of entrapped drug," PharmTech, vol. 8, no. 1, pp.106-113, 2015.

[62] W. Zhan, and X. Y. Xu, "A mathematical model for thermosensitive liposomal delivery of Doxorubicin to solid tumour," Journal of Drug Delivery, 2013.

[63] T. Tagami, M.J. Ernsting, and S.D. Li, "Optimization of a novel and improved thermosensitive liposome formulated with DPPC and a Brij surfactant using a robust in vitro system," Journal of Controlled Release, vol. 154, no. 3, pp. 290-297, 2011.

[64] H. Fujii, K. Noda, Y. Asami, A. Kuroda, M. Sakata and A. Tokida, "Increase in bioluminescence intensity of firefly luciferase using genetic modification," Analytical biochemistry, vol. 366, no. 2, pp. 131-136, 2007.

[65] P.D. Marcato, "Pharmacokinetics and pharmacodynamics of nanomaterials," in Nanotoxicology. New York, NY, USA: Springer, 2014, pp. 97-110. 